\title{
CONVOLUTION PROPERTY AND EXPONENTIAL BOUNDS FOR SYMMETRIC MONOTONE DENSITIES
}

\author{
Claude Lefìvre ${ }^{1}$ And Sergey UteV ${ }^{2}$
}

\begin{abstract}
Our first theorem states that the convolution of two symmetric densities which are $k$-monotone on $(0, \infty)$ is again (symmetric) $k$-monotone provided $0<k \leq 1$. We then apply this result, together with an extremality approach, to derive sharp moment and exponential bounds for distributions having such shape constrained densities.
\end{abstract}

Mathematics Subject Classification. 60E10, 60E15.

Received June 15, 2011. Revised April 13, 2012.

\section{MAIN RESUlts}

\subsection{Symmetric monotone densities}

A function $f$ on $(0, \infty)$ is completely monotone if $(-1)^{j} f^{(j)}(x) \geq 0$ for $j=0,1, \ldots$ By Bernstein's theorem, there exists some nondecreasing function $\alpha(y)$ such that $f$ can be represented as

$$
f(x)=\int_{0}^{\infty} \mathrm{e}^{-x y} \mathrm{~d} \alpha(y), \quad x>0 .
$$

Functions $f$ that are monotone of finite order $k$ on $(0, \infty)$ have been studied by Williamson [22], Lévy [14] and Gneiting [8]. $f$ is 1-monotone means that it is nonnegative and nonincreasing. For an integer $k \geq 2, f$ is $k$-monotone if $(-1)^{j} f^{(j)}(x)$ is nonegative, nonincreasing and convex for $j=0,1, \ldots, k-2$. An analogous representation for $f$ still holds, namely

$$
f(x)=\int_{0}^{\infty}(1-x y)_{+}^{k-1} \mathrm{~d} \alpha(y), \quad x>0,
$$

for some nondecreasing function $\alpha(y)$ bounded at 0 . The case of a real order $k \geq 1$ was also discussed by Williamson [22]. By extension, $f$ is said to be $k$-monotone, $k \geq 1$, if it can be written under the previous integral form.

\footnotetext{
Keywords and phrases. Multiply monotonicity, symmetric densities, unimodality, Wintner's theorem, Bernstein's inequality.

1 Département de Mathématique, Université Libre de Bruxelles, Campus de la Plaine C.P. 210, 1050 Bruxelles, Belgique. clefevre@ulb.ac.be

2 School of Mathematical Sciences, University of Nottingham, University Park, NG7 2RD Nottingham, UK.

sergey.utev@nottingham.ac.uk
} 
Now, let us consider for $f$ a density function on $(0, \infty)$. A general expression for a $k$-monotone density when $k$ is a positive integer was obtained by Lévy [14], Theorem 5; it can also be recovered from Williamson's results. Hereafter, we retain this representation as a definition for any positive order $k$. Specifically, a density $f$ on $(0, \infty)$ is $k$-monotone, $k>0$, if it has the form

$$
f(x)=\int_{0}^{\infty} \frac{k}{y}\left(1-\frac{x}{y}\right)_{+}^{k-1} \mathrm{~d} G(y), \quad x>0,
$$

for some distribution function $G(y)$. In other words, $f$ is $k$-monotone when it can be represented as a scale mixture of beta $(1, k)$ densities (given by $k(1-x)^{k-1}, 0<x<1$ ). For $k=1$, this definition recovers a wellknown fact that nonincreasing densities are in a bijection with scale mixtures of uniform densities [11]. For $k=2$, this is another standard property that nonincreasing convex distributions are necessarily scale mixtures of triangular distributions.

Let $X$ be a random variable having a $k$-monotone density on $(0, \infty)$. By $(1.1)$,

$$
X={ }_{d}\left(1-U^{1 / k}\right) Y,
$$

where $Y$ is a random variable with distribution function $G$, and $U$ is a random variable uniform on $(0,1)$ and independent of $Y$. In the theory of unimodality, $X$ is said to have a beta $(1, k)$-unimodal distribution with mode at 0 (see e.g. the book by Bertin et al. [4], p. 72, where $k$ is a positive real). In the framework of Pakes and Navarro [16], $X$ is viewed as a randomly scaled version of $Y$ with a beta $(1, k)$ scaling factor. Recently, the problem of estimation of a $k$-monotone density has been investigated when $k$ is a positive integer; see e.g. Balabdaoui and Wellner [1].

\subsection{Convolution of symmetric monotone densities: an extension to Wintner's theorem}

The preservation of various properties under summation plays an important role in probability and statistics (see e.g. $[10,20]$ ). So, a natural question is whether the $k$-monotonicity is preserved under convolution.

This topic has been studied for usual unimodal distributions. In general, the convolution of two unimodal distributions is not unimodal. It is easy to see, however, that the convolution of two symmetric uniform distributions is (symmetric) unimodal. A classical theorem by Wintner [23] states that the convolution of two symmetric unimodal distributions is again (symmetric) unimodal (see also [5], p. 13).

For $k$-monotone densities $f$, it follows directly from the representation (1.1) that there exists some positive $\delta$ such that $f(x) \geq \delta$ for $0<x \leq \delta$. This implies, for instance, that the convolution of two exponential densities (which are $\infty$-monotone) is not $k$-monotone for any $k>0$. Now, consider the set of symmetric $k$-monotone densities, i.e. densities symmetric on $\mathbb{R}$ that are $k$-monotone on $(0, \infty)$. The example below illustrates that such densities may not be preserved by convolution.

Example. Let $X_{1}$ and $X_{2}$ be two independent random variables with a Laplace distribution of density $(1 / 2) \exp (-|x|), x \in \mathbb{R}$ (which is symmetric $\infty$-monotone). Putting $S_{X_{1}+X_{2}}(t)=P\left(X_{1}+X_{2}>t\right), t>0$, one gets

$$
S_{X_{1}+X_{2}}(t)=\mathrm{e}^{-t} / 2+t \mathrm{e}^{-t} / 4
$$

hence $S_{X_{1}+X_{2}}^{\prime \prime}(t)=t \mathrm{e}^{-t} / 4>0$ and $S_{X_{1}+X_{2}}^{(3)}(t)=(1-t) \mathrm{e}^{-t} / 4$ is not constant in sign. This means that the convolution is monotone of order 1 (as stated by Wintner's theorem) but not 2 .

Nevertheless, our first theorem in the present paper provides a positive result on the convolution of symmetric monotone densities. This result can be viewed as a generalization of Wintner's theorem for unimodal distributions.

Theorem 1.1. The convolution of two densities that are symmetric $k$-monotone with $0<k \leq 1$ is again (symmetric) k-monotone. 
The proof is given in Section 2.1. It will consist in a careful analysis through a fractional integral operator, with the (evident) preservation of symmetric monotonicity under mixture.

\subsection{Exponential bounds for symmetric monotone densities}

Let $\left\{X_{1}, \ldots, X_{n}\right\}$ be a sequence of i.i.d. random variables having symmetric densities that are $k$-monotone on $(0, \infty)$ with $0<k \leq 1$. By $(1.2)$, the $X_{i}$ 's are distributed as a random variable

$$
X={ }_{d} B\left(1-U^{1 / k}\right) Y
$$

where $B$ is a symmetric random variable on $\{-1,1\}$, independent of $U$ and $Y$. Our purpose is to obtain an upper bound for large deviations of the weighted sums

$$
\sum_{i=1}^{n} a_{i} X_{i}, \text { when } a_{1}, \ldots, a_{n} \geq 0 \text { and } \sum_{i=1}^{n} a_{i}^{2}=1 .
$$

This problem is partly motivated by a work of Barthe and Koldobsky [2] who derived inequalities of this type under concavity properties of the Laplace transform of a probability measure. The approach here is different and relies on sharp moment inequalities.

Theorem 1.2. Assume that $E\left(Y^{2 r}\right)<\infty, r=1,2, \ldots$, and the sequence

$$
(1+2 k+2 r) E\left(Y^{2 r}\right) / E\left(Y^{2 r+2}\right), \quad r=0,1, \ldots,
$$

is nondecreasing in $r$. When $k \leq 1$, then for any $m=1,2, \ldots$,

$$
E\left(\sum_{i=1}^{n} a_{i} X_{i}\right)^{2 m} \leq E\left(n^{-1 / 2} \sum_{i=1}^{n} X_{i}\right)^{2 m} \leq \sigma^{2 m} E\left(\eta^{2 m}\right),
$$

where $\sigma^{2} \equiv \operatorname{Var}(X)$ and $\eta$ is a standard normal random variable. Furthermore, for $x \geq \sigma$,

$$
P\left(\sum_{i=1}^{n} a_{i} X_{i}>x\right) \leq c_{k} \exp \left(-x^{2} / 2 \sigma^{2}\right),
$$

where

$$
c_{k}=\sqrt{e} k^{k}(k+1) /(k+2)^{k+1} .
$$

The proof is given in Section 2.2. We will derive the optimal moment inequalities (1.5) by following an approach developed in Eaton [6] (see e.g. Figiel et al. [7] for an overview on sharp moment inequalities). For the exponential bound (1.6), we will first apply an optimal Markov type inequality which relies on the convolution property of Theorem 1.1. As pointed out in Rio [19], using moment bounds rather than exponential ones (as in the Chernoff bound) gives an increasing flexibility. Then, to obtain the constant $c_{k}$, we will adopt an approach of Hoeffding [9] by identifying the extremal points in the convex set of densities under concern. The latter method is powerful for various problems, e.g. extremal moment inequalities [21] and optimal constants [17], Stein-Chen techniques [13] and actuarial risk theory [12].

\section{PROOFS}

\subsection{Proof of Theorem 1.1}

When $k=1$, this is Wintner's theorem, so assume $k<1$. Let $X_{1}$ and $X_{2}$ be any two independent random variables that have symmetric $k$-monotone densities. By (1.2),

$$
X_{1}={ }_{d}\left(1-U_{1}^{1 / k}\right) Y_{1} \text {, and } X_{2}={ }_{d}\left(1-U_{2}^{1 / k}\right) Y_{2},
$$


where $Y_{1}, Y_{2}, U_{1}, U_{2}$ are independent, $U_{1}, U_{2}$ are $(0,1)$ uniforms and $Y_{1}, Y_{2}$ have symmetric distributions on $\mathbb{R}$. The proof of the theorem is subdivided in two parts: first we will reformulate the problem in a simpler way, then we will use a fractional integral operator.

Reformulation of the problem. As a preliminary, we observe that it suffices to consider $Y_{1}$ as a binary symmetric variable on $\{-1,1\}$ and $Y_{2}$ as a binary symmetric variable on $\{-b, b\}$ with $0<b<1$. Indeed, a simple operation of mixing and scale change then allows us to cover the general case. Moreover, $b$ may be taken $<1$ as the limit of uniformly bounded symmetric $k$-monotone distributions is still $k$-monotone. Evidently, it is equivalent to show that $\left|X_{1}+X_{2}\right|$ has a $k$-monotone density.

By summation and definition of $X_{2}$, for $t>0$,

$$
\begin{aligned}
S_{\left|X_{1}+X_{2}\right|}(t) & =2 P\left(X_{1}+X_{2}>t\right)=\frac{k}{b} \int_{-b}^{b} P\left(X_{1}>t-u\right)\left(1-\frac{|u|}{b}\right)^{k-1} \mathrm{~d} u \\
& =\frac{k}{b} \int_{0}^{b}\left[P\left(X_{1}>t-u\right)+P\left(X_{1}>t+u\right)\right]\left(1-\frac{u}{b}\right)^{k-1} \mathrm{~d} u,
\end{aligned}
$$

where $0<b<1$. Thus, again by mixing, it suffices to prove that for any $u \in(0, b)$, the function

$$
Q(t)=P\left(X_{1}>t-u\right)+P\left(X_{1}>t+u\right), \quad t \geq 0,
$$

is $k+1$-monotone (in the usual sense).

Let us derive another representation for $Q$. By definition of $X_{1}$, if $t \geq u$,

$$
Q(t)=(1 / 2)[1-(t-u)]_{+}^{k}+(1 / 2)[1-(t+u)]_{+}^{k},
$$

while if $0<t<u$, as $P\left(X_{1}>t-u\right)=1-P\left(X_{1}>u-t\right)$,

$$
Q(t)=1-(1 / 2)[1-(u-t)]_{+}^{k}+(1 / 2)[1-(t+u)]_{+}^{k} .
$$

Both cases can be combined to give

$$
Q(t)=(1 / 2)\left\{[1-(t-u)]_{+}^{k}+[1-(t+u)]_{+}^{k}\right\}+I_{(0 \leq t<u)}\left\{1-(1 / 2)[1-(u-t)]_{+}^{k}-(1 / 2)[1+(u-t)]_{+}^{k}\right\} .
$$

Note that for $0 \leq t<u$ with $u \leq b<1$, necessarily $0<u-t<1$, so that the two indices + in the second term $\{\ldots\}$ of the right-hand side are superfluous. Finally, we introduce a binary symmetric variable $B$ on $\{-1,1\}$, so that $Q(t)$ is rewritten as

$$
\begin{aligned}
Q(t) & =E(1-t+u B)_{+}^{k}+I_{(0 \leq t<u)}\left\{1-E[1+(u-t) B]^{k}\right\} \\
& =Q_{1}(t)+Q_{2}(t) \text { say, } \quad t \geq 0 .
\end{aligned}
$$

Now, one can express $Q_{1}$ under the form

$$
Q_{1}(t)=\alpha \int_{0}^{\infty}\left(1-\frac{t}{x}\right)_{+}^{k} \mathrm{~d} \mu_{1}(x), \quad t \geq 0,
$$

where $\mu_{1}$ is a binary probability measure on $\{1-u, 1+u\}$ having point masses $(1-u)^{k} / 2 \alpha$ and $(1+u)^{k} / 2 \alpha$ respectively, with $\alpha \equiv E(1+u B)^{k}$. Thus, $Q_{1} / \alpha$ is a $k+1$-monotone function. Furthermore, we are going to derive for $Q_{2}$ the following representation:

$$
Q_{2}(t)=\int_{0}^{\infty}(x-t)_{+}^{k} \nu_{2}(x) \mathrm{d} x, \quad t \geq 0
$$

where $\nu_{2}$ is a continuous nonnegative function on its support $(0, u)$. Thus, up to a positive constant, $Q_{2}$ too is a $k+1$-monotone function. So, it remains to prove (2.3). 
Analysis through a fractional integral operator. For that, we will use the concept of right-sided RiemannLiouville integral of order $k>0$ (see e.g. Podlubny [18]). This integral of a suitable function $f$ on $(0, \infty)$ is defined by

$$
I^{k} f(t)=\frac{1}{\Gamma(k)} \int_{0}^{\infty}(x-t)_{+}^{k-1} f(x) \mathrm{d} x, \quad t>0 .
$$

The left-sided version of this integral was used by Williamson [22] to characterize monotonicity of real order $k \geq 1$. A key property is that the family of operators satisfies the semi-group property:

$$
I^{k}\left[I^{l} f(t)\right]=I^{k+l} f(t), \quad k, l>0 .
$$

Let us go back to (2.3), and define

$$
J(t)=\int_{0}^{\infty}(x-t)_{+}^{-k} Q_{2}(x) \mathrm{d} x, \quad t \geq 0 .
$$

Notice that $Q_{2}(t)=\Gamma(k+1) I^{k+1} \nu_{2}(t)$, so that by $(2.5)($ as $0<k<1)$,

$$
I^{1-k} Q_{2}(t)=\Gamma(k+1) I^{1-k} I^{1+k} \nu_{2}(t)=\Gamma(1+k) I^{2} \nu_{2}(t) .
$$

Thus, (2.6) can be rewritten as

$$
\begin{aligned}
J(t) & =\Gamma(1-k) I^{1-k} Q_{2}(t)=\Gamma(1-k) \Gamma(1+k) I^{2} \nu_{2}(t) \\
& =\Gamma(1-k) \Gamma(1+k) \int_{0}^{\infty}(x-t)_{+} \nu_{2}(x) \mathrm{d} x, \quad t \geq 0 .
\end{aligned}
$$

By definition, $Q_{2}(t)=0$ for $t \geq u$ (see (2.2)), hence the integrals in (2.6), (2.7) are restricted to the interval $[0, u]$. In particular, $J(t)=0$ for $t \geq u$.

Now, we are going to prove that this function $J$ is continuously differentiable, nonnegative, nonincreasing and convex on $(0, \infty)$. Therefore, $J$ admits the representation

$$
J(t)=\int_{0}^{\infty}(x-t)_{+} J^{\prime \prime}(x) \mathrm{d} x, \quad t \geq 0,
$$

which corresponds to the right-hand side of (2.7) with $\nu_{2}(x)=J^{\prime \prime}(x) / \Gamma(1-k) \Gamma(1+k)$.

We may focus on the case $t \in(0, u)$. By changing the variable $x$ to $z=(u-x) /(u-t)$,

$$
\begin{aligned}
J(t) & =\int_{t}^{u}(x-t)^{-k}\left\{1-E[1+(u-x) B]^{k}\right\} \mathrm{d} x \\
& =(u-t)^{1-k} \int_{0}^{1}(1-z)^{-k}\left\{1-E[1+(u-t) z B]^{k}\right\} \mathrm{d} z \\
& =(u-t)^{1-k} \Lambda(t) \text { say. }
\end{aligned}
$$

Note that $J$ is continuous on $(0, u)$ and $\lim _{t \uparrow u} J(t)=0$. Thus, $J$ is continuous on $(0, \infty)$. 
Put $w \equiv(u-t) z$ for $t \in(0, u)$, so that $w \in(0,1)$. The function $R(y)=(1+y)^{k}$ being concave on $|y|<1$, Jensen's inequality implies

$$
E[1+(u-t) z B]^{k}=E R(w B) \leq R[w E(B)]=1,
$$

which shows that $\Lambda$, and thus $J$, is nonnegative.

Consider $J^{\prime}$ on $(0, u)$. From $(2.8)$, we get

$$
\Lambda^{\prime}(t)=k \int_{0}^{1} z(1-z)^{-k} E\left\{B[1+(u-t) z B]^{k-1}\right\} \mathrm{d} z
$$

and as $k-1<0$, using again the notation $w$ inside the term $E\{\ldots\}$,

$$
2 E\left[B(1+w B)^{k-1}\right]=(1+w)^{k-1}-(1-w)^{k-1}<0,
$$

so that $\Lambda^{\prime}$, and thus $J^{\prime}$, is negative. Moreover, $\lim _{t \uparrow u} J^{\prime}(t)=0$. Indeed,

$$
J^{\prime}(t)=(u-t)^{-k} \int_{0}^{1}(1-z)^{-k} \phi[(u-t) z] \mathrm{d} z,
$$

where $\phi[(u-t) z]=\phi(w)$ is given by

$$
\phi(w)=k E\left[w B(1+w B)^{k-1}\right]+(k-1)\left[1-E(1+w B)^{k}\right] .
$$

Note that $w \downarrow 0$ as $t \uparrow u$, uniformly over $z \in[0,1]$. Applying Taylor's expansion, we see that

$$
|\phi(w)| \leq c_{b}|w| \text { for }|w| \leq b<1,
$$

for some positive constant $c_{b}$. Thus, $J^{\prime}$ is continuous on $(0, \infty)$.

Let us examine $J^{\prime \prime}$ on $(0, u)$. From $(2.8)$,

$$
J^{\prime \prime}(t)=k(1-k)(u-t)^{-k-1} \int_{0}^{1}(1-z)^{-k} \psi[(u-t) z] \mathrm{d} z,
$$

where $\psi[(u-t) z]=\psi(w)$ is given by

$$
\begin{aligned}
\psi(w) & =-\left[1-E(1+w B)^{k}\right]-2 E\left[w B(1+w B)^{k-1}\right]+E\left[w^{2}(1+w B)^{k-2}\right] \\
& =-1+H(w) \text { say. }
\end{aligned}
$$

So, $J^{\prime \prime}$ is continuous on $(0, u)$. Moreover, $H$ can be rewritten as

$$
\begin{aligned}
H(w) & =E\left\{(1+w B)^{k-2}\left[(1+w B)^{2}-2 w B(1+w B)+w^{2}\right]\right\} \\
& =E(1+w B)^{k-2},
\end{aligned}
$$

since $B^{2}=1$. As $Z(y) \equiv(1+y)^{k-2}$ is convex on $|y|<1$, by Jensen's inequality

$$
H(w)=E Z(w B) \geq Z[w E(B)]=1,
$$

which implies that $\psi$, and thus $J^{\prime \prime}$, is nonnegative. Thus, $J$ being continuously differentiable nonincreasing on $(0, \infty), J$ is also convex on $(0, \infty)$. 
Remark 2.1. Although related to unimodality, multiple monotonicity is a quite different concept. In particular, one can construct a density $f$ that is $k$-monotone and has infinitely many modes. Consider the successive intervals $\Delta_{i}=(4 \pi(i-1), 4 \pi i], i=1,2, \ldots$, and a function $h$ given by $h(0)=0$ and $h(x)=\left(1 / 2^{i}\right) g[x-4 \pi(i-$ 1)], $x \in \Delta_{i}$, where

$$
g(t)= \begin{cases}\theta \sin (t), & 0 \leq t \leq 2 \pi \\ \sin (t / 2), & 2 \pi \leq t \leq 4 \pi\end{cases}
$$

for some parameter $\theta \in(0,1]$. Then, define $f$ by stipulating that $f^{\prime}=\operatorname{ch}(x), x \in(0, \infty)$, where $c$ is a normalizing constant. Clearly, $f$ has local maxima at points $4 \pi i, i \geq 1$. Now, by adapting the method of proof followed above, one can show that $f$ is a $k$-monotone density for all $k \in(0,1)$.

\subsection{Proof of Theorem 1.2}

For the moment inequalities (1.5), we will exploit the assumption of symmetric $k$-unimodality for $0<k \leq 1$ together with the condition (1.4). For the exponential bounds (1.6), we will also apply the convolution property of Theorem 1.1.

Moment inequalities. To begin with, let us recall the notions of majorization and Schur-concavity (see e.g. the book by Marshall and Olkin [15]). Let $\left(a_{1}, \ldots, a_{n}\right)$ and $\left(b_{1}, \ldots, b_{n}\right)$ be two real vectors whose terms are ranged in decreasing order and are of equal sums. The vector $\left(a_{1}, \ldots, a_{n}\right)$ is majorized (denoted $\preceq$ ) by $\left(b_{1}, \ldots, b_{n}\right)$ if $a_{1} \leq b_{1}, a_{1}+a_{2} \leq b_{1}+b_{2}, \ldots, a_{1}+\ldots+a_{n-1} \leq b_{1}+\ldots+b_{n-1}$, with $a_{1}+\ldots+a_{n}=b_{1}+\ldots+b_{n}$. A real-valued function $f\left(x_{1}, \ldots, x_{n}\right)$ is Schur-concave if $\left(a_{1}, \ldots, a_{n}\right) \preceq\left(b_{1}, \ldots, b_{n}\right)$ implies $f\left(a_{1}, \ldots, a_{n}\right) \geq f\left(b_{1}, \ldots, b_{n}\right)$.

Now, consider the function $s\left(a_{1}, \ldots, a_{n}\right)=E\left(\sum_{i=1}^{n} \sqrt{a}_{i} X_{i}\right)^{2 m}$, for any $m=1,2, \ldots$ As the $X_{i}$ 's are symmetric random variables with finite moments, it is known that $s\left(a_{1}, \ldots, a_{n}\right)$ is a Schur-concave function if the sequence

$$
\psi_{r} \equiv E\left(X^{2 r}\right) E\left(\eta^{2 r+2}\right) / E\left(X^{2 r+2}\right) E\left(\eta^{2 r}\right), \quad r=0,1, \ldots,
$$

is nondecreasing in $r$ (Marshall and Olkin [15], pp. 362-363). This condition is well satisfied in the case (1.3) with $0<k \leq 1$. Indeed, since $E\left(\eta^{2 r}\right)=(2 r) ! / r ! 2^{r}$, and

$$
E\left(1-U^{1 / k}\right)^{s}=\Gamma(s+1) \Gamma(k+1) / \Gamma(s+k+1), \quad s \geq 0,
$$

$\psi_{r}$ is given here by

$$
\psi_{r}=\left(1-t_{r}\right)(1+2 k+2 r) E\left(Y^{2 r}\right) / E\left(Y^{2 r+2}\right),
$$

where $t_{r}=k(1-k) /(1+2 k+2 r)(2 r+2)$. Thus, by the assumptions (1.4) and $0<k \leq 1, \psi_{r}$ is nondecreasing in $r$.

The two inequalities in (1.5) follow directly from the Schur-concavity of $s\left(a_{1}, \ldots, a_{n}\right)$. For the former, it suffices to use the evident majorization $(1 / n, \ldots, 1 / n) \preceq\left(a_{1}^{2}, \ldots, a_{n}^{2}\right)$. For the latter, working with vectors of larger length $n+N$ for $N \geq 1$, we proceed similarly with $[1 /(n+N), \ldots, 1 /(n+N)] \preceq[1 / n, \ldots, 1 / n, 0, \ldots, 0]$, and we then take the limit $N \rightarrow \infty$ to apply a CLT.

Note that alternatively, a comparison with the Gaussian variable could be made by the extremal approach in Figiel et al. [7].

Exponential bounds. A key point is that as $0<k \leq 1$, the random variable $\sum_{i=1}^{n} a_{i} X_{i}$ has also a symmetric $k$-monotone density. So, denote by $\mathcal{K}_{k, s}$ the set of distributions with symmetric $k$-monotone densities and finite moments of order $s>0$. Applying a Markov type argument, we write

$$
P\left(\left|\sum_{i=1}^{n} a_{i} X_{i}\right|>x\right) \leq A(k, s) \frac{E\left|\sum_{i=1}^{n} a_{i} X_{i}\right|^{s}}{x^{s}}, \quad x>0
$$


where

$$
\begin{aligned}
A(k, s) & =\sup _{F_{X} \in \mathcal{K}_{k, s}} \sup _{x>0} \frac{x^{s} P(|X| \geq x)}{E|X|^{s}} \\
& =\sup _{F_{X} \in \mathcal{K}_{k, s}} g\left(F_{X}\right) \text { say. }
\end{aligned}
$$

Observe that the functional $g$ is scale-invariant (i.e. $g\left(F_{a X}\right)=g\left(F_{X}\right)$ for any a) and weakly convex (i.e. $g\left(p_{1} F_{X_{1}}+p_{2} F_{X_{2}}\right) \leq \max \left[g\left(F_{X_{1}}\right), g\left(F_{X_{2}}\right)\right]$ for any $p_{1}, p_{2}>0$ with $\left.p_{1}+p_{2}=1\right)$. These properties together with the representation (1.3) of $X$ imply that

$$
A(k, s)=g\left(F_{1-U^{1 / k}}\right)=\sup _{x \in(0,1)} \frac{x^{s} P\left(1-U^{1 / k} \geq x\right)}{E\left(1-U^{1 / k}\right)^{s}} .
$$

Since the function $x^{s} P\left(1-U^{1 / k} \geq x\right)$ has its supremum at point $x=s /(s+k)$, we then get

$$
A(k, s)=\frac{\Gamma(s+k+1)}{\Gamma(s+1) \Gamma(k+1)}\left(\frac{s}{s+k}\right)^{s}\left(\frac{k}{s+k}\right)^{k} .
$$

Let us now take $s=2 m$ in (2.9). We first note that $A(k, s)$ is decreasing in $s$ since, putting $\delta=k /(s-1)>0$ and $y=1 /(s+k)<1$,

$$
[A(k, s) / A(k, s-1)]^{1 / k}=(1-y) \exp [(1 / \delta) \log (1+\delta y)] \leq(1-y) \exp (y) \leq 1 .
$$

Hence, we deduce that

$$
P\left(\sum_{i=1}^{n} a_{i} X_{i}>x\right) \leq \frac{A(k, 2)}{2} \inf _{m=1,2, \ldots} \frac{E\left(\sum_{i=1}^{n} a_{i} X_{i}\right)^{2 m}}{x^{2 m}}, \quad x>0,
$$

where $A(k, 2)=2 k^{k}(k+1) /(k+2)^{k+1}$. In addition, since $x^{2} \geq \sigma^{2}$ by assumption, the infimum in (2.12) may be taken over all $m=0,1,2, \ldots$

We are ready to insert in (2.12) the upper moment bound given in (1.5). This yields

$$
P\left(\sum_{i=1}^{n} a_{i} X_{i}>x\right) \leq \frac{A(k, 2)}{2} \inf _{m=0,1,2, \ldots} \frac{E(\eta)^{2 m}}{(x / \sigma)^{2 m}} .
$$

Moreover, a bound in Rio [19], page 40 (Thm. 2.4) gives

$$
\inf _{m=0,1,2, \ldots} \frac{E(\eta)^{2 m}}{(x / \sigma)^{2 m}} \leq \sqrt{e} \exp \left(-x^{2} / 2 \sigma^{2}\right),
$$

hence the desired exponential bound (1.6).

Remark 2.2. Assumption (1.4) is a form of sub-Gaussian condition convenient to apply the majorization technique to the present case. Note that (1.4) depends on $k$; in fact, if it is satisfied for an order $\tilde{k}>k$, then it is true for $k$ too. Furthermore, one can check that the bounding constant $c_{k}$ in (1.6) is decreasing in the order $k$.

By comparison with Barthe and Koldobsky [2], our method based on moment inequalities is applicable to distributions that do not have a tractable Laplace transform. When the $X_{i}$ 's are uniformly distributed on $[-1 / 2,1 / 2]$, i.e. if $k=1$ and $Y=1 / 2$ a.s., then the upper bound in (1.6) becomes $(2 \sqrt{e} / 9) \exp \left(-6 x^{2}\right)$; the factor $2 \sqrt{e} / 9$ allows us to improve a basic inequality in Section 5 of their paper (when $x \geq \sigma=1 / \sqrt{12}$ ). For $k=0$ and $k=1$, the assumption (1.4) corresponds to the condition given in their Theorem 7, point (ii). When the $X_{i}$ 's have a symmetric Bernoulli law, i.e. if $k=0$ and $Y=1$ a.s., then (1.6) holds with $c_{0}=\sqrt{e} / 2$, which becomes a Bernstein inequality with the improved constant by Rio [19]. On another hand, it could be interesting to see whether a similar approach is applicable to the models studied in Barthe et al. [3]. 
To close, let us point out that (2.10) combined with (2.11) provides us also with a Markov type inequality within the set of distributions $\mathcal{K}_{k, s}$ for $0 \leq k \leq 1$. For instance, if $k=1$ and $s=2, A(1,2)=4 / 9$ and $(2.10)$ becomes the Gauss inequality. If $k=0, A(0, s)=1$ and (2.10) gives a classical Markov inequality. For unimodal distributions, such inequalities can be found e.g. in Dharmadhikari and Joag-dev [5], Section 1.5.

Acknowledgements. We are grateful to two referees and the associate editor for very helpful remarks and suggestions. C. Lefèvre thanks the Banque Nationale de Belgique for its support. The research of S. Utev was supported by the Fonds National de la Recherche Scientifique Belge.

\section{REFERENCES}

[1] F. Balabdaoui and J.A. Wellner, Estimation of a $k$-monotone density: limit distribution theory and the spline connection. Ann. Stat. 35 (2007) 2536-2564.

[2] F. Barthe and A. Koldobsky, Extremal slabs in the cube and the Laplace transform. Adv. Math. 174 (2003) 89-114.

[3] F. Barthe, F. Gamboa, L. Lozada-Chang and A. Rouault, Generalized Dirichlet distributions on the ball and moments. ALEA Latin Amer. J. Probab. Math. Stat. 7 (2010) 319-340.

[4] E.M.J. Bertin, I. Cuculescu and R. Theodorescu, Unimodality of Probability Measures. Kluwer, Dordrecht (1997).

[5] S. Dharmadhikari and K. Joag-dev, Unimodality, Convexity, and Applications. Academic, San Diego (1988).

[6] M.L. Eaton, A note on symmetric Bernoulli random variables. Ann. Math. Stat. 41 (1970) 1223-1226.

[7] T. Figiel, P. Hitczenko, W.B. Johnson, G. Schechtman and J. Zinn, Extremal properties of Rademacher functions with applications to the Khintchine and Rosenthal inequalities. Trans. Amer. Math. Soc. 349 (1997) 997-1027.

[8] T. Gneiting, Radial positive definite functions generated by Euclid's hat. J. Multiv. Anal. 69 (1999) 88-119.

[9] W. Hoeffding, The extrema of the expected value of a function of independent random variables. Ann. Math. Stat. 26 (1955) $268-275$.

[10] O. Johnson and C. Goldschmidt, Preservation of log-concavity on summation. ESAIM: PS 10 (2005) $206-215$.

[11] A.Y. Khintchine, On unimodal distributions. Izv. Nauchno- Issled. Inst. Mat. Mech. Tomsk. Gos. Univ. 2 (1938) 1-7 (in Russian).

[12] C. Lefèvre and S. Loisel, On multiply monotone distributions, discrete or continuous, with applications. Working paper, ISFA, Université de Lyon 1 (2011).

[13] C. Lefèvre and S. Utev, Exact norms of a Stein-type operator and associated stochastic orderings. Probab. Theory Relat. Fields 127 (2003) 353-366.

[14] P. Lévy, Extensions d'un théorème de D. Dugué et M. Girault. Wahrscheinlichkeitstheorie 1 (1962) $159-173$.

[15] A.W. Marshall and I. Olkin, Inequalities: Theory of Majorization and its Applications. Academic, New York (1979).

[16] A.G. Pakes and J. Navarro, Distributional characterizations through scaling relations. Aust. N. Z. J. Stat. 49 (2007) $115-135$.

[17] I. Pinelis, Toward the best constant factor for the Rademacher-Gaussian tail comparison. ESAIM: PS 11 (2007) $412-426$.

[18] I. Podlubny, Fractional Differential Equations. Academic, San Diego (1999).

[19] E. Rio, Théorie Asymptotique des Processus Aléatoires Faiblement Dépendants. Springer, Berlin (2000).

[20] M. Shaked and J.G. Shanthikumar, Stochatic Orders. Springer, New York (2007).

[21] S.A. Utev, Extremal problems in moment inequalities, in Limit Theorems of Probability Theory of Trudy Inst. Mat., vol. 5. Nauka Sibirsk. Otdel., Novosibirsk. (1985) 56-75 (in Russian).

[22] R.E. Williamson, Multiply monotone functions and their Laplace transforms. Duke Math. J. 23 (1956) $189-207$.

[23] A. Wintner, On a class of Fourier transforms. Amer. J. Math. 58 (1936) 45-90. 